\title{
Output Feedback Control for Asymptotic Stabilization of Spacecraft with Input Saturation
}

\author{
Chutiphon Pukdeboon \\ Nonlinear Dynamic Analysis Research Center, Department of Mathematics, Faculty of Applied Science, \\ King Mongkut's University of Technology North Bangkok, Bangkok, Thailand \\ Correspondence should be addressed to Chutiphon Pukdeboon; chutiphon.p@sci.kmutnb.ac.th
}

Received 19 May 2017; Accepted 11 September 2017; Published 1 November 2017

Academic Editor: Manuel Pineda-Sanchez

Copyright (C) 2017 Chutiphon Pukdeboon. This is an open access article distributed under the Creative Commons Attribution License, which permits unrestricted use, distribution, and reproduction in any medium, provided the original work is properly cited.

\begin{abstract}
This paper investigates the attitude stabilization problem of rigid spacecraft subject to actuator constraints, external disturbances, and attitude measurements only. An output feedback control framework with input saturation is proposed to solve this problem. The general saturation function is utilized in the proposed controller design and a unified control method is developed for the asymptotic stabilization of rigid spacecraft without velocity measurements. Asymptotic stability is proven by Lyapunov stability theory. Moreover, a new nonlinear disturbance observer is designed to compensate for external disturbances. Then, a composite controller is presented by combining a unified saturated output feedback control with a nonlinear disturbance observer. Desirable features of the proposed control scheme include the intuitive structure, robustness against external disturbances, avoidance of model information and velocity measurements, and ability to ensure that the actuator constraints are not violated. Finally, numerical simulations have been carried out to verify the effectiveness of the proposed control method.
\end{abstract}

\section{Introduction}

The stabilization of spacecraft has been studied intensively in the last few decades. Several control results that stabilize the attitude of the spacecraft have been proposed in the literature. For example, Egeland and Godhavn [1] presented a passivitybased adaptive control method for rigid spacecraft. In [2], a velocity-free controller is developed to achieve the asymptotic attitude stabilization. Su and Zheng [3] designed two nonlinear PD controllers to obtain finite-time stabilization based on Rodrigues parameter and modified Rodrigues parameter, respectively. Joshi et al. [4] presented a nonlinear control law based on unit quaternion feedback for asymptotic stability of spacecraft which is robust to modeling errors. Zhao and Jia [5] designed a continuous control law to ensure finite-time convergence for a class of stochastic spacecraft systems. In [6$8]$, several optimal control and $H$-infinity control approaches have been proposed to achieve the stabilization of the rigid spacecraft in the presence of external disturbance and model uncertainties. Sliding mode control has attracted extensive attention owing to its robustness with respect to external disturbances and model uncertainties. This control method has been applied to attitude stabilization of spacecraft in $[9,10]$.

However, all these aforementioned works assume that the actuator can generate any required control torque. This is quite conservative. In practice, when the requested control torque exceeds the maximum value that the actuator can supply, the performance of the controlled system cannot be guaranteed and this may cause instability [11-13]. In order to overcome this drawback, several approaches that consider actuator constraints have been proposed. More specifically, Wallsgrove and Akella [14] used a smooth variable structure attitude stabilization control scheme. In [15], a saturated controller is designed based on the backstepping control technique. Recently, Su and Zheng [16] present a unified saturated PD control framework to achieve asymptotic stabilization. They used the generalized saturated function to obtain the desired results. In [17], a saturated attitude controller for spacecraft subject to actuator constraints and bounded disturbances has been proposed. Finite-time attitude stabilization has been studied in [18]. Using the theory 
of homogeneous system, finite-time stability of the attitude control system can be guaranteed.

While these controllers consider the actuator constraints, they assume that all the states can be measured and used in the controller. In practice, while the velocity can be measured by the sensor, it is influenced easily by the noise. Thus, the accuracy cannot be guaranteed and the performance will be degraded. This makes a velocity-free controller more desirable [19]. Recognizing this limitation, velocity-free control methods for rigid spacecraft have been presented. For example, Akella et al. [20] utilized a filter to deal with the attitude variables and thus a velocity-free controller was proposed for the stabilization of spacecraft in the presence of torque-magnitude and rate-saturation limits. By using an angular-velocity observer, $\mathrm{Hu}$ and Zhang [21] proposed a bounded output feedback controller to stabilize a spacecraft system. Su and Zheng [22] developed two velocityfree controllers to deal with asymptotic stabilization based on Rodrigues parameter and modified Rodrigues parameter, respectively. follows.

The major contributions of this paper are summarized as

(1) A unified saturated output feedback (USOF) controller is developed for asymptotic stabilization of spacecraft. The velocity is replaced with a filter. The control torque can be constrained less than the maximum value that the actuator can supply by selecting suitable control gains.

(2) To the best of the authors' knowledge, the results presented in this work are the first attempt to design a composite controller combining a USOF controller with a nonlinear disturbance observer (NDO).

Advantages of the proposed approach include the intuitive structure, easy implementation, avoidance of model information and velocity measurements, robustness against external disturbances, and avoidance of violation of actuation limits.

The remainder of this paper is organized as follows. The problem of designing a saturated output feedback controller for rigid spacecraft is formulated in Section 2. Section 3 presents the controller design and stability analysis. In Section 4, an illustrative example is provided to verify the improved performance of the proposed approach. Finally, some conclusions are given in Section 5.

The following notations are used throughout this paper. $\lambda_{\max }(A)$ denotes the maximum eigenvalue of matrix $A$. $\|y\|$ indicates the Euclidean norm of a vector $y$. The norm of matrix $A$ is defined as the corresponding induced norm which can be written as $\|A\|=\sqrt{\lambda_{\max }\left(A^{T} A\right)}$, and $I_{3}$ represents the 3-by-3 identity.

\section{Problem Statements}

The unit quaternion is used to describe the kinematics of rigid spacecraft because it is free of singularity. The unit quaternion is a vector defined as $Q=\left[\begin{array}{ll}q^{T} & q_{4}\end{array}\right]^{T}$, where $q=\left[\begin{array}{lll}q_{1} & q_{2} & q_{3}\end{array}\right]^{T}$ is the vector part and $q_{4}$ is the scalar part. They are subject to norm constraint $q^{T} q+q_{4}^{2}=1$. by [2]

The kinematic model of rigid spacecraft can be described

$$
\begin{aligned}
& \dot{q}=T(Q) \omega, \\
& \dot{q}=-\frac{1}{2} q^{T} \omega,
\end{aligned}
$$

where $\omega=\left[\begin{array}{lll}\omega_{1} & \omega_{2} & \omega_{3}\end{array}\right]^{T}$ denotes the angular vector of the spacecraft, $T(Q)=(1 / 2)\left(q_{4} I_{3}+q^{x}\right)$, and $a^{\times}$is a skewsymmetric matrix which denotes an operator on any vector $a=\left[\begin{array}{lll}a_{1} & a_{2} & a_{3}\end{array}\right]^{T}$ such that

$$
a^{\times}=\left[\begin{array}{ccc}
0 & -a_{3} & a_{2} \\
a_{3} & 0 & -a_{1} \\
-a_{2} & a_{1} & 0
\end{array}\right] .
$$

The dynamic equation for spacecraft is governed by [2]

$$
J \dot{\omega}=-\omega^{\times} J \omega+u+d,
$$

where $J \in \Re^{3 \times 3}$ is the symmetry positive definite constant inertia matrix, $u=\left[\begin{array}{lll}u_{1} & u_{2} & u_{3}\end{array}\right]^{T}$ is the control input, and $d=$ $\left[\begin{array}{lll}d_{1} & d_{2} & d_{3}\end{array}\right]^{T}$ is the external disturbance.

We assume that each actuator has a known maximum torque, $u_{i, \max }, i=1,2,3$. We also assume that only the attitude can be measured and utilized in the feedback control. The control objective is to design a unified saturated output feedback controller for the spacecraft to achieve asymptotic stabilization and satisfy

$$
\left|u_{i}\right| \leq u_{i, \max }
$$

where $u_{i}$ denotes the $i$ th control torque of the $i$ th actuator.

\section{Controller Design}

3.1. Control Formulation. Before the control design, the definition for the generalized saturation function given in [23] is restated.

Definition 1 (generalized saturation function [23]). Given a positive constant $M$, a continuous function $s: \mathfrak{R} \rightarrow \mathfrak{R}$ is said to be a generalized saturated function if it satisfies

(a) $y s(y)>0, \forall y \neq 0$;

(b) $|s(y)| \leq M, \forall y \in \Re$.

For our control development, the following lemma will be exploited.

Lemma 2. The matrix $T(Q)$ defined by (1) has the norm constraint that $\|T(Q)\|=1 / 2$. 
Proof. By the definition of $T(Q)$ that $T(Q)=(1 / 2)\left(q_{4} I_{3}+q^{\times}\right)$, it follows that

$$
\begin{gathered}
T(Q)^{T} T(Q)=\frac{1}{4}\left[\begin{array}{ccc}
q_{4} & q_{3} & -q_{2} \\
-q_{3} & q_{4} & q_{1} \\
q_{2} & -q_{1} & q_{4}
\end{array}\right]\left[\begin{array}{ccc}
q_{4} & -q_{3} & q_{2} \\
q_{3} & q_{4} & -q_{1} \\
-q_{2} & q_{1} & q_{4}
\end{array}\right] \\
=\frac{1}{4}\left[\begin{array}{ccc}
q_{2}^{2}+q_{3}^{2}+q_{4}^{2} & -q_{1} q_{2} & -q_{1} q_{3} \\
-q_{1} q_{2} & q_{1}^{2}+q_{3}^{2}+q_{4}^{2} & -q_{2} q_{3} \\
-q_{1} q_{3} & -q_{2} q_{3} & q_{1}^{2}+q_{2}^{2}+q_{4}^{2}
\end{array}\right] .
\end{gathered}
$$

By calculating $q q^{T}$ and using the identity $q_{1}^{2}+q_{2}^{2}+q_{3}^{2}+q_{4}^{2}=$ 1 , we can conclude that

$$
T(Q)^{T} T(Q)=\frac{1}{4}\left(I_{3}-q q^{T}\right) .
$$

Using the fact that the eigenvalues of $q q^{T}$ are $q^{T} q, 0,0$, we have

$$
\begin{aligned}
\lambda_{\max }\left(T(Q)^{T} T(Q)\right) & =\max \left\{\frac{1}{4}\left(I_{3}-q^{T} q\right), \frac{1}{4}, \frac{1}{4}\right\} \\
& =\frac{1}{4},
\end{aligned}
$$

where $\lambda_{\max }(A)$ denotes the maximum eigenvalue of matrix A.

As a consequence,

$$
\|T(Q)\|=\frac{1}{2} .
$$

This completes the proof.

Now, the unified saturated output feedback controller is designed in the presence of actuator constraints and attitude measurements only. We propose the USOF controller for asymptotic stabilization of rigid spacecraft as

$$
\begin{aligned}
u & =-2 T^{T}(Q) K_{1} q-2 T^{T}(Q) K_{2} S(v), \\
v & =q_{c}+B q, \\
\dot{q}_{c} & =-A v
\end{aligned}
$$

where $K_{1}=\operatorname{diag}\left\{k_{11}, k_{12}, k_{13}\right\}, K_{2}=\operatorname{diag}\left\{k_{21}, k_{22}, k_{23}\right\}$, $A=\operatorname{diag}\left\{a_{1}, a_{2}, a_{3}\right\}, B=\operatorname{diag}\left\{b_{1}, b_{2}, b_{3}\right\}, k_{i j}>0, a_{j}>$ $0, b_{j}>0, i=1,2, j=1,2,3$. In (11), $q_{c}$ is an auxiliary variable vector, and $S(v)$ is a vector saturation function defined as

$$
S(v)=\left[\begin{array}{llll}
s\left(v_{1}\right) & s\left(v_{2}\right) & s\left(v_{3}\right)
\end{array}\right]^{T} .
$$

From the formulation of the controller and Lemma 2 and using Definition 1 of the generalized saturation function with $M=1$, we can conclude that

$$
\left|u_{i}\right| \leq k_{1 i}+k_{2 i}, \quad i=1,2,3 .
$$

As a consequence, by selecting suitable control gains to satisfy

$$
k_{1 i}+k_{2 i}<u_{i, \max }
$$

this ensures that the actuator constraints will not be violated.
3.2. Stability Analysis. The first main result of this paper is given in Theorem 3.

Theorem 3. Consider the spacecraft dynamic and kinematic equations (1), (2), and (4) subject to actuator constraints (5) and suppose that the disturbance term $d$ in (4) is not taken into account. The proposed saturated output feedback controller given by (10)-(12) ensures asymptotic stabilization.

Proof. Consider the following Lyapunov function candidate:

$$
V=\frac{1}{2} \omega^{T} J \omega+q^{T} K_{1} q+2 K_{2} B^{-1} \int_{0}^{\nu} S(\tau) d \tau,
$$

where $\int_{0}^{v} S(\tau) d \tau=\sum_{i=1}^{3} \int_{0}^{v_{i}} s(\tau) d \tau$.

Using Definition 1, one has

$$
\int_{0}^{v} S(\tau) d \tau>0, \quad \forall v \neq 0 \in \mathfrak{R}^{3} .
$$

As a result, one can conclude that the proposed Lyapunov function given by (10) is radially unbounded positive definite with respect to $\omega, q, v$.

Differentiating (16) with respect to time yields

$$
\dot{V}=\omega^{T} J \dot{\omega}+2 \dot{q}^{T} K_{1} q+2 S^{T}(v) K_{2} B^{-1} \dot{v} .
$$

Substituting (10)-(12) into (18) and using the property of skew-symmetric matrix $\omega^{\times}$, it follows that

$$
\begin{aligned}
\dot{V}= & \omega^{T}\left[-2 T^{T}(q) K_{1} q-2 T^{T}(q) K_{2} S(v)\right]+2 \dot{q}^{T} K_{1} q \\
& +2 S^{T}(v) K_{2} B^{-1}[-A v+B \dot{q}] \\
= & -2 S^{T}(v) K_{2} B^{-1} A v .
\end{aligned}
$$

Using the fact given by (a) in Definition 1, it is evident that $\dot{V}$ is a negative semidefinite function. Moreover, $\dot{V}=0$ implies that $v=0$. Hence, by LaSalle's invariance principle [24], the origin is asymptotically stable. This completes the proof.

Remark 4. The proposed controller can be considered as a nonlinear proportional-derivative (PD) control method. This controller has the advantages of the intuitive structure, the absence of modeling information and velocity measurements, and the ability to guarantee that the actuator constraints are not violated. Moreover, the stability of the closed loop with the proposed controller does not depend on a specific saturation function. This offers an additional appealing property that it may obtain an improved performance by choosing the saturation function freely.

Remark 5. In (19), since $\dot{V}$ is negative semidefinite, the asymptotic stabilization cannot be achieved by using only the Lyapunov analysis. In this paper, LaSalle's invariance principle [24] is introduced to ensure the asymptotic stabilization of the spacecraft.

\section{Disturbance Observer Design}

The control development in Section 3 is properly designed when the total disturbance $d(t)$ is not taken into account. 
If the total disturbance $d(t)$ has an effect on the spacecraft system, then the stability of the closed-loop system is not guaranteed. In this section, a nonlinear disturbance observer is designed to compensate for the total disturbance $d(t)$. It is clear that the specific information of the total disturbance $d(t)$ is difficult to obtain in practical engineering owing to the complicated structure of disturbance. However, it is reasonable to assume that $d(t)$ and $\dot{d}(t)$ are bounded.

Thus, there exist constants $\delta>0$ and $\gamma \geq 0$ such that

$$
\begin{gathered}
\|d(t)\| \leq \delta, \\
\|\dot{d}(t)\| \leq \gamma,
\end{gathered}
$$

where $\|d\|$ indicates the Euclidean norm of a vector $d$.

Next, the second main result of this paper is presented in Theorem 6 .

Theorem 6. Consider the spacecraft dynamic and kinematic equations (1), (2), and (4) subject to actuator constraints (5) and the disturbance term $d$ in (4). When a nonlinear disturbance observer is designed in the form of (21), there exists a constant $\beta>0$ such that the observer error dynamics are bounded and stable.

$$
\begin{aligned}
& \dot{\hat{d}}=\sigma+\beta J \omega, \\
& \dot{\sigma}=-\beta \sigma-\beta\left(\beta J \omega-\omega^{\times} J \omega+u\right),
\end{aligned}
$$

where $\widehat{d}$ is the estimate of $d$ and $\sigma$ is the auxiliary variable.

Proof. Define $\widetilde{d}=\widehat{d}-d$ as the estimate error. The error dynamics of NDO can be derived from (4) and (21) as

$$
\begin{aligned}
\dot{\vec{d}} & =\dot{\vec{d}}-\dot{d}=\dot{\sigma}+\beta J \dot{\omega}-\dot{d} \\
& =-\beta \sigma-\beta\left(\beta J \omega-\omega^{\times} J \omega+u\right)+\beta J \dot{\omega}-\dot{d} \\
& =-\beta(\sigma+\beta J \omega)+\beta\left(J \dot{\omega}+\omega^{\times} J \omega-u\right)-\dot{d} \\
& =-\beta \widehat{d}+\beta d-\dot{d}=-\beta \widetilde{d}-\dot{d} .
\end{aligned}
$$

Then, the Lyapunov function $W$ is chosen as

$$
W=\frac{1}{2} \tilde{d}^{T} \widetilde{d}
$$

Differentiating $W$ with respect to time, one obtains

$$
\begin{aligned}
\dot{W} & =\tilde{d}^{T}(-\beta \tilde{d}-\dot{d}) \leq-\beta\|\tilde{d}\|^{2}+\|\tilde{d}\|\|\dot{d}\| \\
& \leq-\beta\|\tilde{d}\|^{2}+\gamma\|\tilde{d}\| .
\end{aligned}
$$

According to (24), $\dot{W} \leq-\beta\|\widetilde{d}\|^{2}$ can be obtained if $\|\widetilde{d}\|>\gamma / \beta$. Then, the decrease of $W$ forces the system state into $\|\widetilde{d}\| \leq$ $\gamma / \beta$. This implies that the estimate error $\tilde{d}$ converges into a small bounded region $\gamma / \beta$ by selecting a suitable observer gain $\beta$. The proof is completed.
Next, a composite controller is designed by combining the USOF controller with NDO. The proposed NDO-USOF controller is given as

$$
u=-2 T^{T}(Q) K_{1} q-2 T^{T}(Q) K_{2} S(v)-\widehat{d}
$$

The asymptotic stabilization of the closed-loop system under controller (22) is proven in the following theorem.

Theorem 7. Consider the spacecraft dynamic and kinematic equations (1), (2), and (4) subject to actuator constraints (5) and the disturbance term $d$ in (4). The NDO-USOF controller given by (25) ensures that all state variables in (1), (2), and (4) are uniformly ultimately bounded.

Proof. Consider the Lyapunov function defined in (16). One can see that $\dot{V}$ becomes

$$
\begin{aligned}
\dot{V}= & \omega^{T}\left[-2 T^{T}(q) K_{1} q-2 T^{T}(q) K_{2} S(v)-\widehat{d}+d\right] \\
& +2 \dot{q}^{T} K_{1} q+2 S^{T}(v) K_{2} B^{-1}[-A v+B \dot{q}] \\
\leq & -2 S^{T}(v) K_{2} B^{-1} A v+\|\omega\|\|\tilde{d}\| .
\end{aligned}
$$

According to Theorem 6, by selecting a suitable observer gain $\beta$, the estimate error $\widetilde{d}$ converges into a residual set of zero. With bounded motion around the origin, the trajectory of the closed-loop system eventually converges into a region of the origin. This implies that state variables in (1), (2), and (4) are uniformly ultimately bounded. This completes the proof.

Remark 8. Different from NDO-based controllers in [25, 26], this study considers multiple disturbances for three-axis attitude stabilization of rigid spacecraft. The proposed NDOUSOF controller is more robust than the traditional linear feedback controllers for a system with strong nonlinearities and multiple disturbances.

\section{Simulation Results}

The spacecraft used in [6] is selected to accomplish the comparison. The system parameter in [6] is given by $J=$ $\operatorname{diag}\{10,15,20\} \mathrm{kg}-\mathrm{m}^{2}$ and the angular velocity is initialized as zero. The initial attitude parameter is $q(0)=$ $\left[\begin{array}{llll}0.4646 & 0.1928 & 0.8047 & 0.3153\end{array}\right]^{T}$. The maximum control torques are supposed to be $u_{\max }=\left[\begin{array}{lll}5 & 5 & 5\end{array}\right]^{T} \mathrm{Nm}$. The sampling period is $T=1 \mathrm{~ms}$.

The generalized saturation function used in [27] is selected to show the effectiveness and improved performance of the proposed control.

$$
s(y)= \begin{cases}|y|^{\alpha} \operatorname{sgn}(y), & \delta<|y| \leq 1 \\ \delta^{\alpha-1} y, & |y| \leq \delta \\ \operatorname{sgn}(y), & |y|>1,\end{cases}
$$

where $\alpha>0, \delta<1$, and $\operatorname{sgn}(y)$ is the standard signum function. 

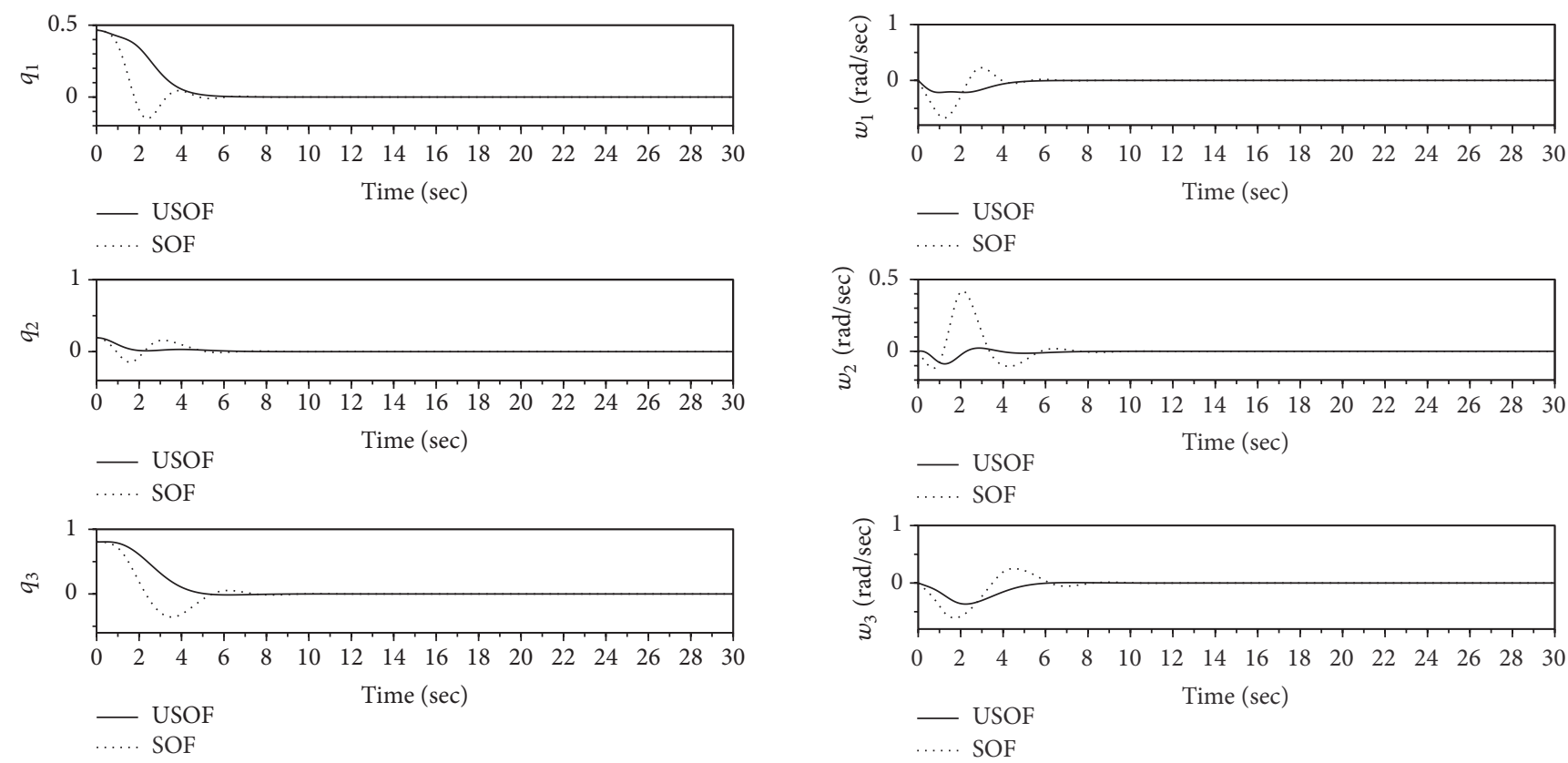

FIGURE 1: Quaternion parameters without disturbances.

The saturated output feedback (SOF) controller proposed in [20] is chosen to verify the improved performance of the proposed controller. For the stabilization case, the SOF control law of [20] is given by

$$
\begin{aligned}
u & =-k_{p} q-\frac{k_{d}}{\sqrt{3}}\left(q_{4} I_{3}+q^{\times}\right) \tanh \left(\lambda\left(q-k^{2} z\right)\right), \\
\dot{z} & =q-k^{2} z, \\
z(0) & =\frac{1}{k^{2}} q(0),
\end{aligned}
$$

where $k_{p}, k_{d}, \lambda \in \Re$ are positive design constants, $z \in \mathfrak{R}^{3}$ is an auxiliary variable, and $\tanh (\cdot) \in \mathfrak{R}^{3}$ is the vector function composed of the hyperbolic function.

All the controller gains are selected by trial and error until a better convergence time is obtained. According to the control constraints (5), the control gains are selected as $K_{1}=\operatorname{diag}(12,14,8), \quad K_{2}=\operatorname{diag}(22,20,30), \quad A=\operatorname{diag}(2,2$, $2), B=\operatorname{diag}(0.2,0.2,0.2), \alpha=0.5$, and $\delta=0.01$. The parameters in controllers (10) and (25) are selected as $k_{p}=$ $5, k_{d}=29, k=1.5$, and $\lambda=2$. First, the simulations without disturbance are performed under the USOF controller (10). The quaternion parameters and angular velocities are shown in Figures 1 and 2. For USOF, the quaternion parameters and angular velocities converge faster to zero and achieve more accurate positioning performance than the SOF control of [20]. The control torque comparison is given by Figure 3. Responses of control torques obtained by USOF have a more rapid convergence rate than SOF control of [20].

After that, the simulations in the presence of disturbances have been conducted under the NDO-USOF controller (25). The external disturbance including constant disturbance and
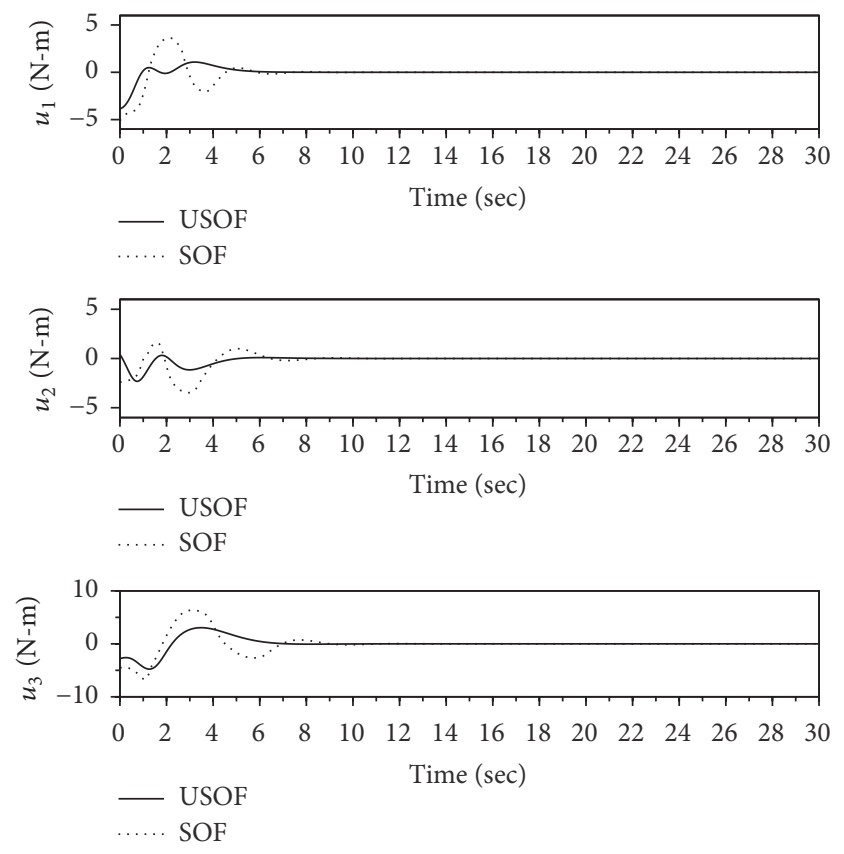

FIGURE 3: Control torque without disturbances.

periodic disturbance of different frequencies is described as

$$
d=0.01 \times\left[\begin{array}{c}
3 \cos (t)-10+4 \sin (0.3 t) \\
3 \cos (0.5 t)+15-1.5 \sin (0.2 t) \\
3 \sin (t)+10+8 \sin (0.4 t)
\end{array}\right] \mathrm{Nm}
$$



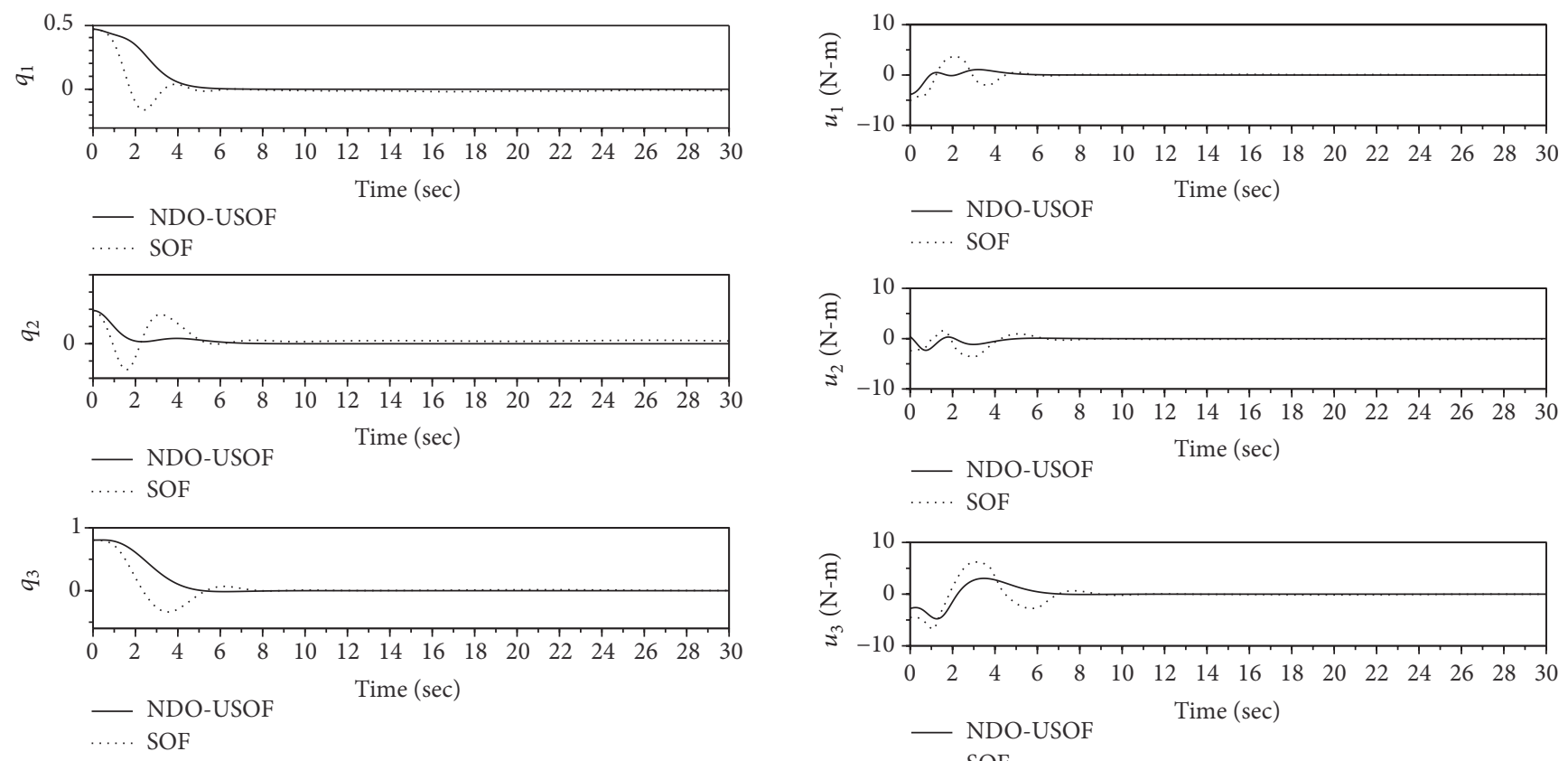

FIGURE 4: Quaternion parameters with disturbances.
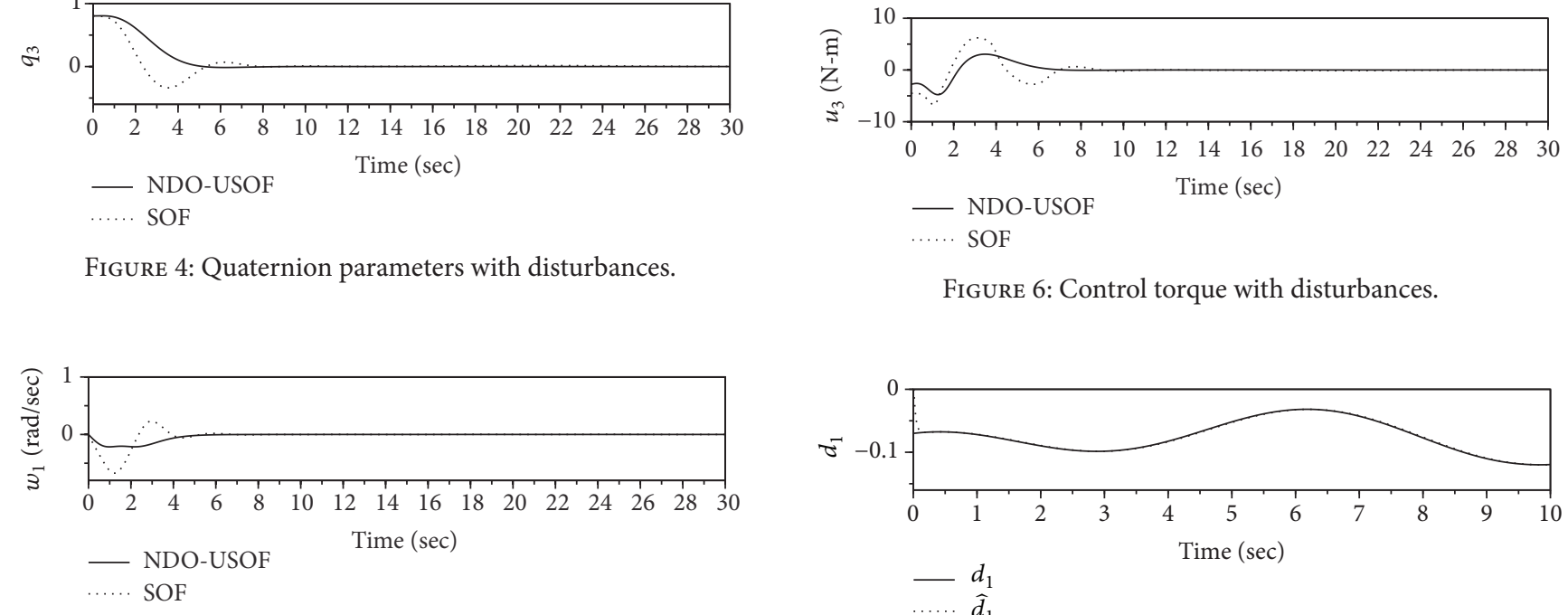

FIGURE 6: Control torque with disturbances.
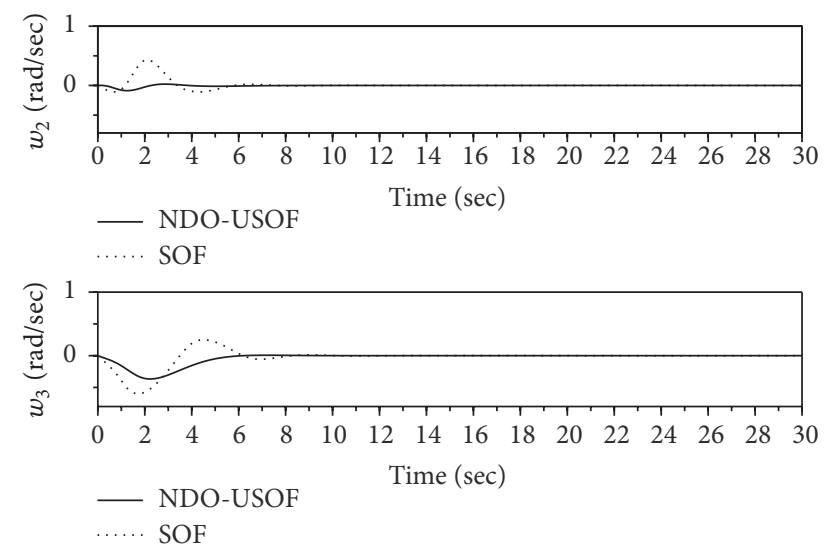

FIGURE 5: Angular velocity with disturbances.

The simulation results are given in Figures 4-7. From Figures 4 and 5, one can see that NDO-USOF provides much better responses of quaternion parameters and angular velocities. Specifically, Figure 4 shows that, for SOF, responses of quaternion parameters cannot be stabilized to zero. As shown in Figure 6, the control torques remain uniform with the requested torque constraints. Figure 7 shows that NDO gives the estimate disturbance $\widehat{d}$ which converges very fast to the true disturbance $d$.
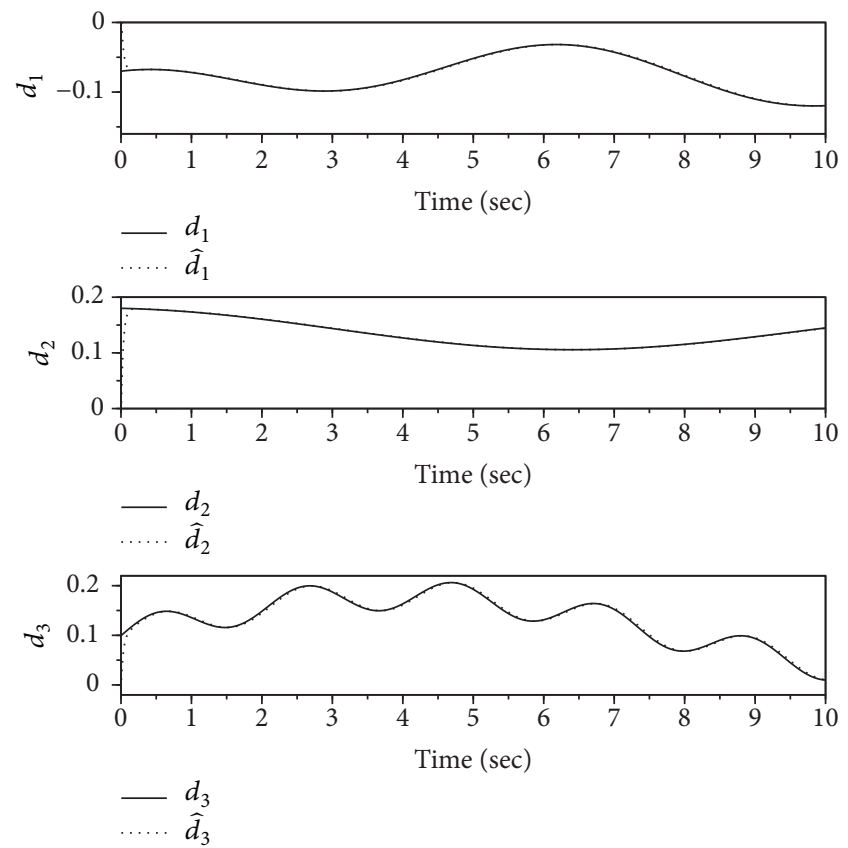

FIGURE 7: Disturbance estimates.

\section{Conclusion}

A unified saturation output feedback controller for asymptotic stabilization of rigid spacecraft with external disturbances and actuator saturation has been proposed. Using the linear filter driven by the attitude variable, velocity measurements are not required. Asymptotic stability has been proven by Lyapunov's direct method. Moreover, a nonlinear disturbance observer is designed to compensate for external 
disturbance. Then, a composite controller is developed by combining a unified saturated output feedback control with nonlinear disturbance observer. Advantages of the proposed control framework are the intuitive structure, the absence of model information and velocity measurements, avoidance of violation of actuation limits, and robustness against external disturbances. Simulations have verified the effectiveness and improved performance of the proposed controller.

\section{Conflicts of Interest}

The author declares that there are no conflicts of interest regarding the publication of this paper.

\section{Acknowledgments}

This research was funded by King Mongkut's University of Technology North Bangkok (Contract no. KMUTNB-60ART-51).

\section{References}

[1] O. Egeland and J.-M. Godhavn, "Passivity-based adaptive attitude control of a rigid spacecraft," IEEE Transactions on Automatic Control, vol. 39, no. 4, pp. 842-846, 1994.

[2] F. Lizarralde and J. T. Wen, "Attitude control without angular velocity measurement: a passivity approach," IEEE Transactions on Automatic Control, vol. 41, no. 3, pp. 468-472, 1996.

[3] Y. Su and C. Zheng, "Simple nonlinear proportional-derivative control for global finite-time stabilization of spacecraft," Journal of Guidance, Control, and Dynamics, vol. 38, no. 1, pp. 173-177, 2015.

[4] S. M. Joshi, A. G. Kelkar, and J. T. Wen, "Robust attitude stabilization of spacecraft using nonlinear quaternion feedback," IEEETransactions on Automatic Control, vol. 40, no. 10, pp. 1800-1803, 1995.

[5] L. Zhao and Y. Jia, "Finite-time attitude stabilisation for a class of stochastic spacecraft systems," IET Control Theory \& Applications, vol. 9, no. 8, pp. 1320-1327, 2015.

[6] M. Krstic and P. Tsiotras, "Inverse optimal stabilization of a rigid spacecraft," IEEE Transactions on Automatic Control, vol. 44, no. 5, pp. 1042-1049, 1999.

[7] C. Pukdeboon and A. S. Zinober, "Control Lyapunov function optimal sliding mode controllers for attitude tracking of spacecraft," Journal of The Franklin Institute, vol. 349, no. 2, pp. 456475, 2012.

[8] W. Kang, "Nonlinear Ho control and its application to rigid spacecraft," Institute of Electrical and Electronics Engineers Transactions on Automatic Control, vol. 40, no. 7, pp. 1281-1285, 1995.

[9] Q. Shen, D. Wang, S. Zhu, and E. K. Poh, "Integral-type sliding mode fault-tolerant control for attitude stabilization of spacecraft," IEEE Transactions on Control Systems Technology, vol. 23, no. 3, pp. 1131-1138, 2015.

[10] C. Pukdeboon and P. Siricharuanun, "Nonsingular terminal sliding mode based finite-time control for spacecraft attitude tracking," International Journal of Control, Automation, and Systems, vol. 12, no. 3, pp. 530-540, 2014.
[11] D. S. Bernstein and A. N. Michel, "A chronological bibliography on saturating actuators," International Journal of Robust and Nonlinear Control, vol. 5, no. 5, pp. 375-380, 1995.

[12] N. O. Pérez-Arancibia, T.-C. Tsao, and J. S. Gibson, "Saturationinduced instability and its avoidance in adaptive control of hard disk drives," IEEE Transactions on Control Systems Technology, vol. 18, no. 2, pp. 368-382, 2010.

[13] W. E. Dixon, "Adaptive regulation of amplitude limited robot manipulators with uncertain kinematics and dynamics," IEEE Transactions on Automatic Control, vol. 52, no. 3, pp. 488-493, 2007.

[14] R. J. Wallsgrove and M. R. Akella, "Globally stabilizing saturated attitude control in the presence of bounded unknown disturbances," Journal of Guidance, Control, and Dynamics, vol. 28, no. 5, pp. 957-963, 2005.

[15] I. Ali, G. Radice, and J. Kim, "Backstepping control design with actuator torque bound for spacecraft attitude maneuver," Journal of Guidance, Control, and Dynamics, vol. 33, no. 1, pp. 254-259, 2010.

[16] Y. Su and C. Zheng, "Unified saturated proportional derivative control framework for asymptotic stabilisation of spacecraft," IET Control Theory \& Applications, vol. 10, no. 7, pp. 772-779, 2016.

[17] H. Jinchang and Z. Honghua, "A simple saturated control framework for spacecraft with bounded disturbances," International Journal of Robust and Nonlinear Control, vol. 26, no. 3, pp. 367384, 2016.

[18] H. Gui, L. Jin, and S. Xu, "Simple finite-time attitude stabilization laws for rigid spacecraft with bounded inputs," Aerospace Science and Technology, vol. 42, pp. 176-186, 2015.

[19] A.-M. Zou, "Finite-time output feedback attitude tracking control for rigid spacecraft," IEEE Transactions on Control Systems Technology, vol. 22, no. 1, pp. 338-345, 2014.

[20] M. R. Akella, A. Valdivia, and G. R. Kotamraju, "Velocityfree attitude controllers subject to actuator magnitude and rate saturations," Journal of Guidance, Control, and Dynamics, vol. 28, no. 4, pp. 659-666, 2005.

[21] J. Hu and H. Zhang, "Bounded output feedback of rigid-body attitude via angular velocity observers," Journal of Guidance, Control, and Dynamics, vol. 36, no. 4, pp. 1240-1247, 2013.

[22] Y. Su and C. Zheng, "Velocity-free saturated PD controller for asymptotic stabilization of spacecraft," Aerospace Science and Technology, vol. 39, pp. 6-12, 2014.

[23] A. R. Teel, "Global stabilization and restricted tracking for multiple integrators with bounded controls," Systems \& Control Letters, vol. 18, no. 3, pp. 165-171, 1992.

[24] J. J. E. Slotine and W. Li, Applied Nonlinear Control, PrenticeHall, New Jersey, NJ, USA, 1991.

[25] H. Liu, L. Guo, and Y. Zhang, "Composite attitude control for flexible spacecraft with simultaneous disturbance attenuation and rejection performance," Proceedings of the Institution of Mechanical Engineers, Part I: Journal of Systems and Control Engineering, vol. 226, no. 2, pp. 154-161, 2012.

[26] J. Qiao and L. Guo, "Antidisturbance fault tolerant control of attitude control systems for microsatellite with unknown input delay," Mathematical Problems in Engineering, vol. 2013, Article ID 804754, 9 pages, 2013.

[27] Y. Su and C. Zheng, "A saturated PD plus scheme for asymptotic tracking of robot manipulators," in Proceedings of the 2009 IEEE International Conference on Robotics and Biomimetics (ROBIO '09), pp. 853-858, Guilin, China, December 2009. 


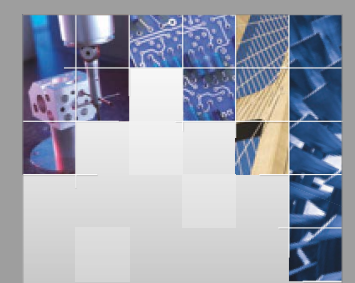

\section{Enfincering}
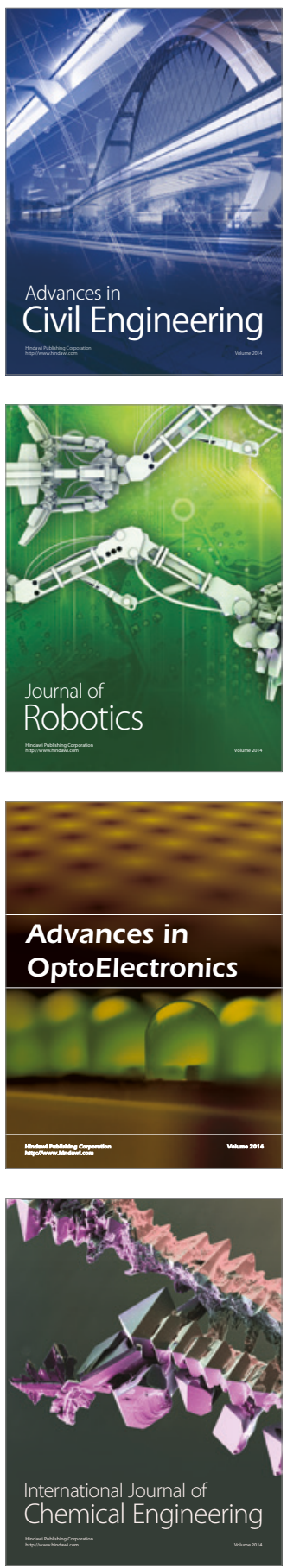

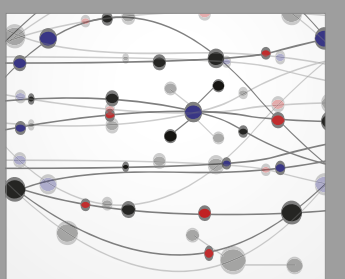

The Scientific World Journal

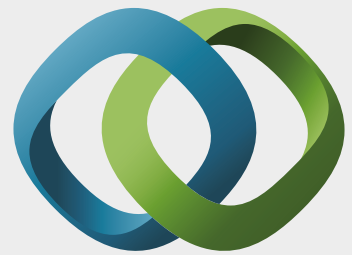

\section{Hindawi}

Submit your manuscripts at

https://www.hindawi.com
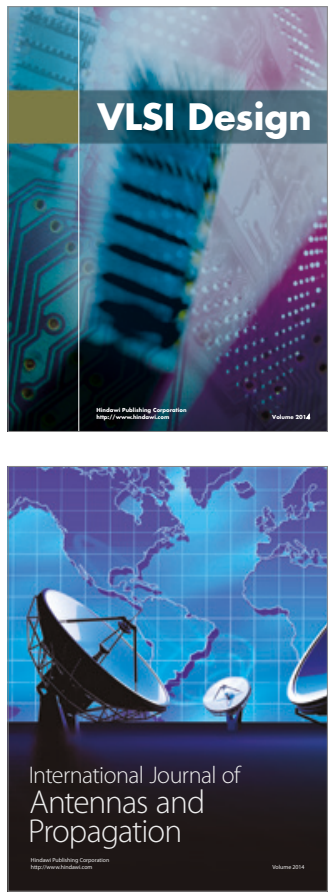

\section{Rotating}

Machinery
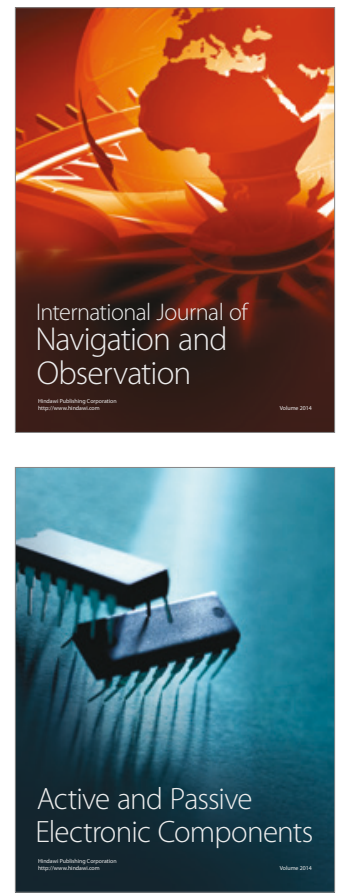
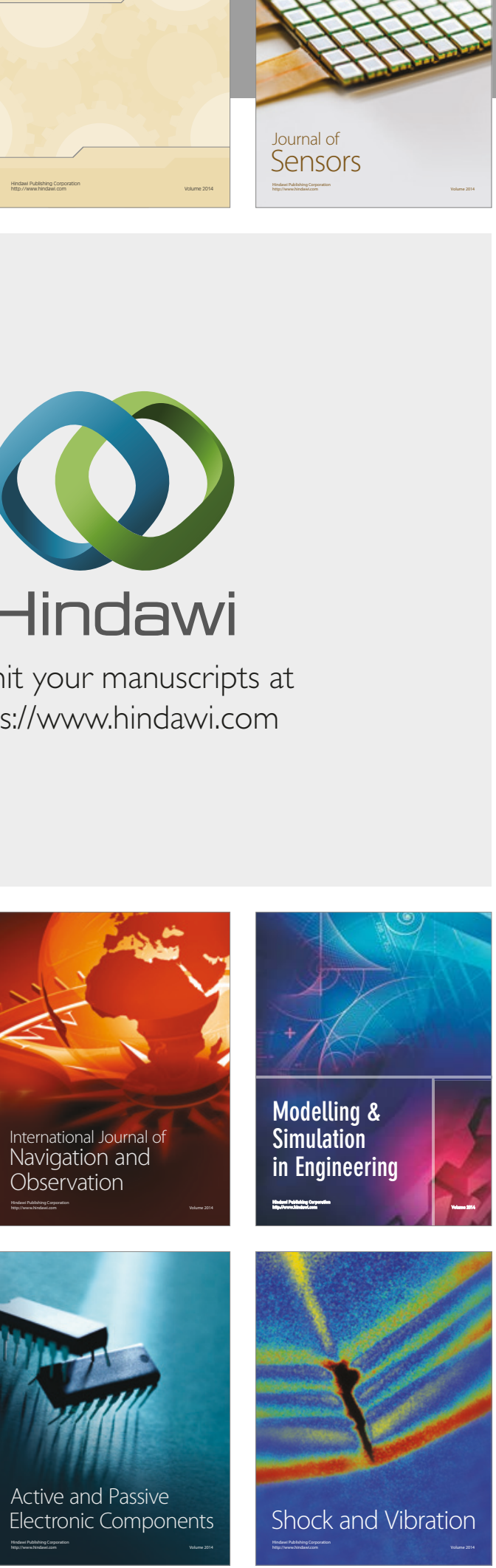
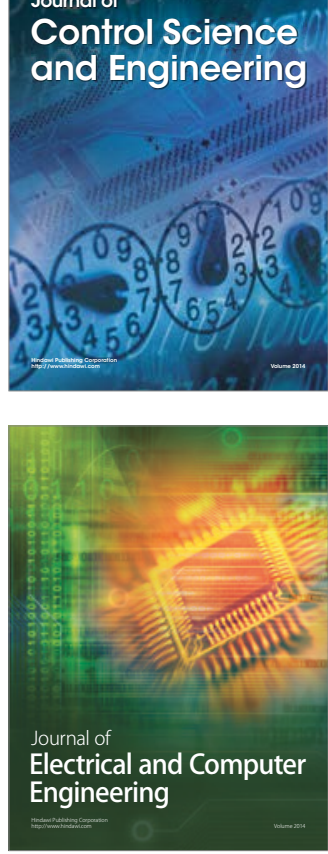

Distributed

Journal of

Control Science

and Engineering
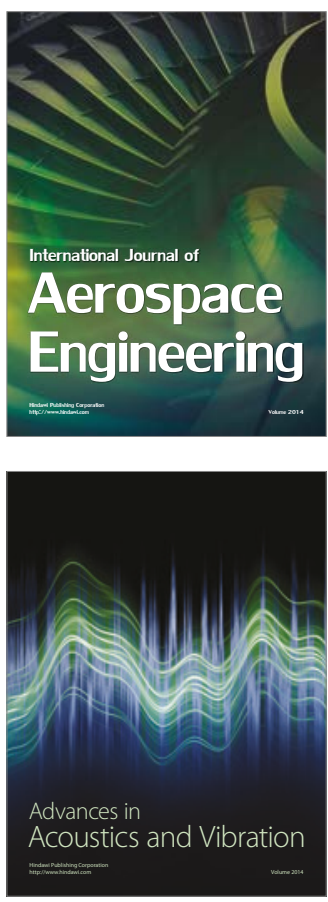

Sensor Networks 